\title{
Evolution of Supercomputers
}

\author{
Garvit Grover \\ The NorthCap University \\ Sector-22, Gurugram \\ Haryana
}

\author{
Geetika Singh \\ The NorthCap University \\ Sector-22, Gurugram \\ Haryana
}

\author{
Jyotsna Singh \\ The NorthCap University \\ Sector-22, Gurugram \\ Haryana
}

\begin{abstract}
This survey paper gives an overview regarding the evolution of Supercomputers. Supercomputing or High-Performance Computing plays ever more important roles in industrial fields as well as in basic research. Super computers are highly developed machines. These are not like computers we use in daily life. We have seen many processors from i5 to i7 to i9 but when we heard about supercomputers we found that these are nothing means these are just a zero level of processors. So, mainly these are the words which we use when defining a supercomputer.
\end{abstract}

\section{General Terms}

Energy, efficiency, development, processors

\section{Keywords}

Supercomputers, petaflops, fluorinet, linux, tiahne

\section{INTRODUCTION}

A commercial computer with vector instruction and pipelined floating-point arithmetic operations is referred to as a supercomputer. [1] These are highly developed mode of computers. On studying the basic concepts of these machines, we found that these are a developed form of a CPU consisting of interconnections between a lot of CPUs. Performance for these computers are measured in flops. Flops stands for floating point operations per second. In my research I found that the fastest supercomputer has a benchmark score of 93 peta flops per second which implies unbelievable speed and most energy efficient among all the supercomputers. Supercomputers were introduced in the 1960s, and for several decades the fastest were made by Seymour Cray at Control Data Corporation (CDC), Cray Research and subsequent companies bearing his name or monogram. The first such machines were highly tuned conventional designs that ran faster than their more general-purpose contemporaries. Through the 1960s, they began to add increasing amounts of parallelism with one to four processors being typical. World's top 500 supercomputers run on Linux-based operating systems. Additional, state of the art research is being conducted in China, United States, European Union, Taiwan and Japan to build even more faster, powerful and technologically superior scale supercomputers. These all are discussed further in this survey paper.

\section{HISTORY TO PRESENT}

The history of supercomputing goes back to the 1960s, with the Atlas at the University of Manchester, the IBM 7030 Stretch and a series of computers at Control Data Corporation (CDC), designed by Seymour Cray. These used innovative designs and parallelism to achieve superior computational peak performance. [2]

The first Atlas was officially commissioned on 7 December 1962 as one of the world's first supercomputers - considered to be the most powerful computer in the world at that time by a considerable margin, and equivalent to four IBM 7094s.
Given that the 6600 outperformed all the other contemporary computers by about 10 times, it was dubbed a supercomputer and defined the supercomputing market, when one hundred computers were sold at $\$ 8$ million each. Cray left CDC in 1972 to form his own company, Cray Research. Four years after leaving CDC, Cray delivered the $80 \mathrm{MHz}$ Cray 1 in 1976, and it became one of the most successful supercomputers in history. The Cray-2 released in 1985 was an 8 processor liquid cooled computer and Fluor inert was pumped through it as it operated. It performed at 1.9giga FLOPS and was the world's second fastest after M-13 supercomputer in Moscow .In 1982, Osaka University's LINKS-1 Computer Graphics System used a massively parallel processing architecture, with 514 microprocessors, including 257 Zilog Z8001 control processors and 257 iAPX $86 / 20$ floating-point processors. It was mainly used for rendering realistic 3D computer graphics. While the supercomputers of the 1980s used only a few processors, in the 1990s, machines with thousands of processors began to appear in Japan and the United States, setting new computational performance records. These are some of the heroes of 90's but when we jumped in 2000 the development increased at very high pace that resulted in high speed supercomputers.

\section{WORKING AND DESIGN}

\subsection{Idea and creating it}

The design for supercomputers was first introduced by Seymour Cray. He introduced the compact design and parallelism in systems to create and develop a new kind of power which is not only bounded up to single core but can go upto multi-cores and hi-tech designs. The first system that was ever created was CDC6600. It was made by joining 10 computers and was used to read and write files. This idea of joining and let the systems work together later resulted in the making of supercomputers. At present these are not less than any superhero, they are a hundred times better than a simple computer. After CDC6600, came CDC7600 and similarly more successors arrived. As after some time, the price, performance and energy efficiency of supercomputers got improvised and then the range of petaFLOPS such as tiahne-I etc. were introduced. This performance in petaFLOPS has a lifecycle or we can say that supercomputers has a lifecycle of 3 years before demanding any new upgrade. As the process becomes common, several special purpose systems have been designed to work on a single problem. For ex: Belle Deep for playing chess, Gravity pipe for astrophysics etc.

\subsection{Energy demand}

Having a great design and processing power, it also needs a lot of energy consumption. For example, tiahne - IA 4.04 megawatts of electricity. It costs around 400 dollars per hour. Even the heat management of these systems costs a lot because they work a lot. 


\subsection{Packing of thousands of processors}

Supercomputers use special techniques for removing the heat from circuits to prevent them from burning up because of their proximity. For ex: CRAY-2 used a liquid cooled by fluorinert regularly under suitable conditions. [3] But as we are going deeper into the technology this issue will also be resolved soon.

\subsection{Materials}

So, to transfer this much of data is not difficult but the difficulty is that we need a material which can work under the heat produced. So, for this copper was chosen to work because copper wire can transfer energy with much higher power densities. So, these all efforts are to maximise the working under the heating issues. The thermal design power of the supercomputer, the amount that the power and cooling infrastructure can handle, is somewhat more than the expected normal power consumption but partially having some lags which are expected to be fixed soon.

\section{SOFTWARE AND SYSTEM MANAGING}

Basically, these systems need as good operating systems as they are themselves. So as an operating system linux is used to operate them because linux is a system that has many capabilities in it. For ex: self-protector from viruses and much more. Linux is mainly used for large scales, if we go on small scales we will find that light weighted kernels such as CNK or $\mathrm{CNL}$ are used. Supercomputers are used in different complex computations. So, it needs a full team of different department, some working on software, some on hardware and others on checking different issues.[4]

Generally, making of supercomputers aims for maximum capability versus capacity ratio. Basically, capability computing is typically thought of as using maximum computing power to solve a single large problem in the least amount of time and these computations are basically in the field of weather simulation, seismic wave analysis, movements of meteoroids etc. And on the other hand, capacity is thought of as using efficient cost-effective computing power to solve a few somewhat large problems or many small problems. So, our basic need is to make a device which has maximum capability is to capacity ratio.

\section{Points scored}

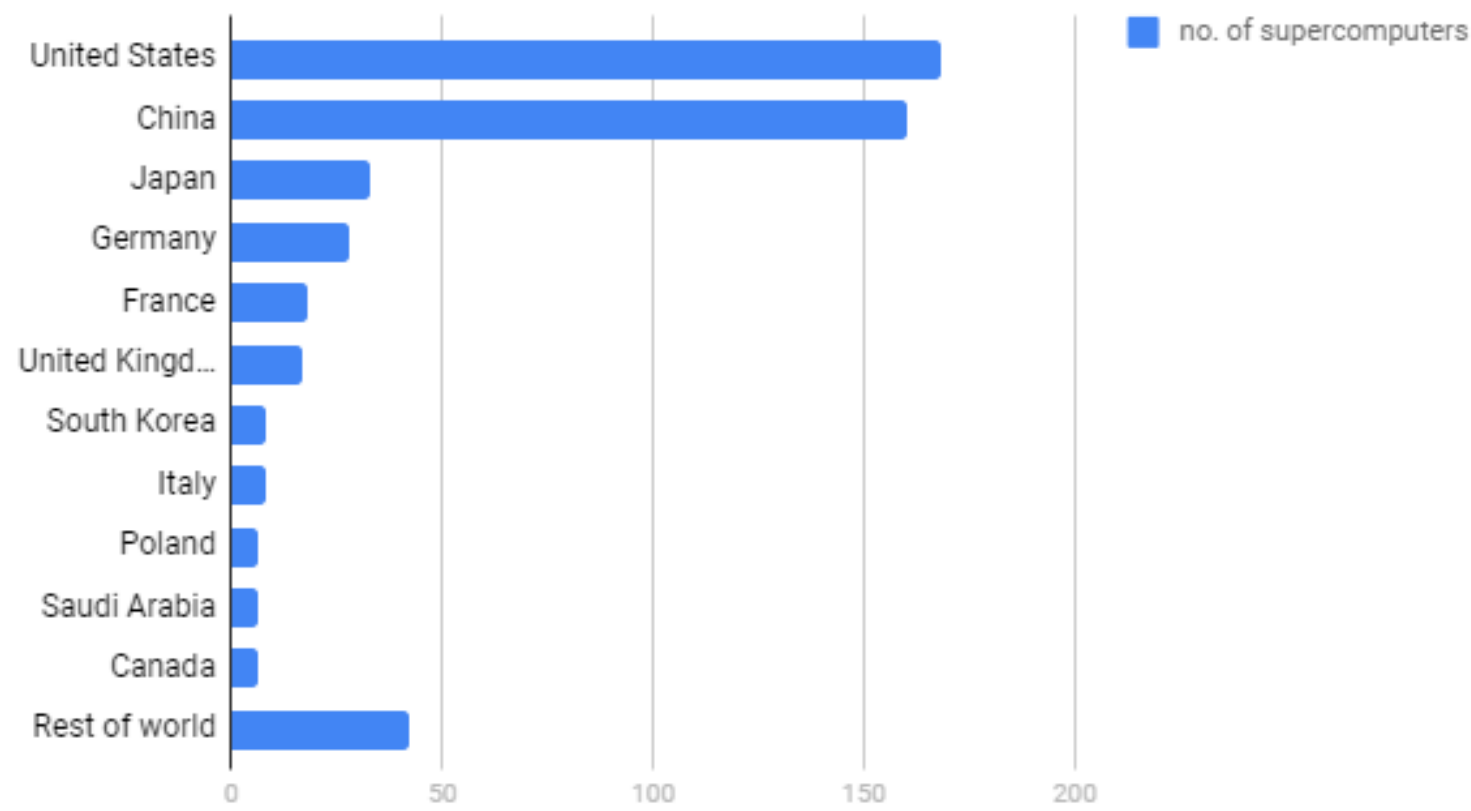

Fig 1:The statistic depicts the distribution of the 500 most powerful supercomputers around the world as of June 2017, by country.

\section{CHINA'S DEVELOPMENT}

If we see, approximately, 10 years before China wasn't even able to get its name in the list of first 500 supercomputers but in the last decade China had worked a lot and is now standing at the top of the world. Today, China has 167 supercomputers in top 500 which is one of the greatest developments world has ever seen. Even USA can't stand in this competition and only has 105 in top 500 and China is leading with a great difference of 62 supercomputers. So, in the past decade China was not only able to come on top but also beats its own supercomputer in terms of energy efficiency and occupies first two positions in supercomputers. They reduced the energy consumption of their supercomputers and was easily able to pass its own system. In the coming years it should be easily able to take a major lead and may even hold up to top 5 positions. [5]

\section{EFFICIENCY}

As is the case with basic computers, the throughput is less i.e. more time is taken to do less work but in case of supercomputers, the time taken to do the same amount of work is less because of vector and parallel pipeline processing in supercomputers. Also, the output of supercomputers is measured in petaflops which is immense. 
Today, Piz Daint is one of the most energy efficient supercomputers with an efficiency level of 3,185.9 megaflops per watt (MFLOPS/W). [6]

\section{DISTRIBUTION OF COMPUTERS COUNTRY WISE}

As of Fig 1, 168 of the top 500 supercomputers were in the United States. [7]

\section{ACKNOWLEDGMENTS}

We thank Ms. Jyotsna Singh, CSE faculty, The NorthCap University(HR.) for comments that greatly improved the manuscript.

\section{REFERENCES}

[1] whatis.techtarget.com en.wikipedia.org/wiki/Supercomputer_operating_systes

[2] en.wikipedia.org, www.americanis.net

[3] gordonbell.azurewebsites.net

[4] insidehpc.com/2016/06/supercomputer-systemsmonitoring-and-management, www.cray.com

[5] www.sciencedirect.com

[6] www.cscs.ch, www.cp2k.org

[7] www.statista.com, www.weforum.org 